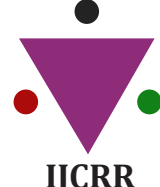

Section: Healthcare

ISI Impact Factor

(2019-20): 1.628

IC Value (2019): 90.81

$\operatorname{SIIF}(\mathbf{2 0 2 0})=7.893$

(c) (i) (3)

Copyright@IJCRR

\title{
The Association between Neutrophil-Lymphocyte Ratio and Clinical Factors Related to Stroke - A Prospective Cohort Study
}

\author{
Badiger $\mathbf{R}^{1}$, Sharma G2*, Chowdary $\mathrm{S}^{2}$, Dulipalli VV', Akki S², Yadav $\mathbf{I}^{2}$, \\ Gandhi $\mathbf{A}^{2}$, Karekar $\mathbf{S}^{2}$
}

'Associate Professor, Department of Medicine, JN Medical College, Belagavi, Karnataka, India; 'Post Graduate, Department of Medicine, JN

Medical College, Belagavi, Karnataka, India.

\section{ABSTRACT}

Introduction and Objectives: Almost 56 million deaths that occur in every year, in that $10.8 \%$ of peoples had died due to stroke. One in five women and one in six men affected by this illness called stroke in their lifetime. Stroke is the leading cause of mortality and disability. NLR and inflammatory marker can be used as a simple and easy marker for prognosis and mortality. The study aims to investigate the role of the NLR ratio in acute ischemic stroke Second foremost reason for death in the global is known as Stroke.

Materials and Methods: Patients with features of acute ischemic stroke were subjected to a detailed history, examination and thorough investigations. The diagnosis was made by clinical history, neurologic examination and neuroimaging. The severity of stroke at the time of admission was classified based on the National Institute of Health Stroke Scale. Modified Ranking Scale used to determine the functional outcome at discharge.

Results: Out of 68 patients enrolled in the study, 13 patients $(19.1 \%)$ had a mild stroke, 45 patients (66\%) were affected by moderate stroke, 6 patients (8.8\%) had moderate to severe stroke, and 8 patients (5.9\%) were severely affected. In the ChiSquare analysis for the association between NLR and MRS, the Pearson chi-square analysis shows significant results (P-value $=0.000<0.001)$

Conclusion: The duration of the hospital stay of the patient depends on NLR. The lifestyle of the patient and diet maintenance also affects the neutrophil and lymphocyte count of the blood (NLR).

Key Words: Acute Ischemic Stroke, Neutrophil Lymphocyte Ratio, Modified Ranking Scale, National Institute of Health Stroke Scale, Lymphocyte Monocyte ratio, Cerebrovascular events

\section{INTRODUCTION}

World Health Organization reported that the fifteen lakh people in global had stroke yearly, among the affected people five lakh decease and five lakh will be eternally handicapped. ${ }^{1,2}$ Stroke is also a leading cause of adult disability. The research reports disability could have been prevented by 80 per cent through proper medical practices. AIS are insufficient arterial supply for cerebral perfusion, because of attenuated or occluded vessels flowing into or inside the brain. It has two types thrombotic and embolic strokes. The blood vessel becomes narrowed and the blood flow to the area beyond is less. Damaged areas of an atherosclerotic plaque lead to a blood clot - a thrombotic stroke. In an embolic stroke, blood clots or debris generated from any other part of the body may block and lead to narrower blood vessels. ${ }^{3,4}$ NLR ratio is the predominant marker for diabetes, renal failure, malnutritional status (mostly chronic), cerebrovascular events, COPD, Alzheimer's and psychiatric illness, metabolic syndrome. Higher blood inflammatory effect on markers like "highly sensitive C-reactive protein (hs-CRP)" is noted as a possible risk for AIS/IHD independently. ${ }^{5,6}$

\section{METHODOLOGY}

\section{SOURCE OF THE STUDY}

The primary data was collected from the patients who were admitted in the wards at KLES Dr. Prabhakar Kore Hospital

\section{Corresponding Author:}

Dr. Sharma Gayatri, Post Graduate, Department of Medicine, JN Medical College 590010, Belagavi, Karnataka, India. Tel: 9916895566; Email: intelllects19@yahoo.com

ISSN: 2231-2196 (Print)

Received: 27.11 .2020
ISSN: 0975-5241 (Online)

Revised: 19.12 .2020
Accepted: 17.04 .2021 
serves also as Medical Research Centre, situated at Belgaum. Informed consent was taken and the database was collected specifically from acute ischemic stroke patients who were admitted to the hospital, within 72 hours of the onset of symptoms, aged above 18 years. The prospective study was for One year ( $1^{\text {st }}$ January 2019 to $31^{\text {st }}$ December 2019)

Ethical clearance was obtained from the institutional ethical committee and informed consent was obtained from each participant before the study. Data from the patients such as demographic factors, comorbidities, duration of hospital stay, day-to-day life activities such as food habits and lifestyle of the patient was obtained. Blood samples were collected once the patient was admitted to finding the neutrophils and lymphocyte counts in their blood. Patients with features of acute ischemic stroke were subjected to a detailed history, examination and thorough investigations.

Clinical history of the patient such as the severity of stroke during admission and the outcome of their health was collected in the database. Neurological examination and Neuroimaging diagnostic methods were used to diagnose the stages of severity of AIS. Based on the NIHSS, the intensity of stroke is classified and functional outcome at the time of discharge is determined using MRS. Patients are followed up in the hospital to retrieve clinical data from the time of admission until discharge. Information collected from the patients is maintained securely as per the informed consent.

The defined co-morbidities include hypertension, type 2 diabetes mellitus, history of CVA, seizure disorder, history of preeclampsia, Ischemic heart disease, depression, hypothyroidism, and rheumatic heart disease. Patients with acute infarction, sub-acute infarction, as well as focal hyperacute infarction were identified and their infracted site was collected to analyse their severity of stroke. During the discharge day of the patient, the number of days the patient got admitted and stayed in the hospital for treatment was collected in the database. Inclusion criteria in this study were the patients with post-acute ischemic stroke admitted before $72 \mathrm{hrs}$ of the onset of symptoms and above 18 years of age. Whereas Patient with a history of more than 3 days of onset of symptoms with a history of infection within 1 week of stroke or 72 hours of the onset of symptoms, with malignancies or haematological symptoms, taking immune suppressant drugs, having a history of recent stroke (for past 6 months) or with previous stroke disability, with the severe pulmonary disorder or chronic renal failure undergoing treatment.

\section{SAMPLING TECHNIQUE}

A prospective sampling technique was used in this study. The sample data was collected from the patients who are admitted to the hospital from the period of 1st January 2019 to 31 st December 2019, specifically those who were admitted for acute ischemic stroke. Among 112 patients admitted at Karnataka Lingayat Education Society's Dr. Prabhakar Kore Hospital \& Medical research centre for acute ischemic stroke, 68 samples were enrolled and the patients, who have chronic renal failure, haemorrhage, malignancies etc. were excluded. Almost $60 \%$ of the samples were selected from the average admission cases in the previous year.

\section{RESULTS}

Among the 68 patients admitted to the hospital, 47 were male and 21 were female. The majority were in the age group of 46-60 years. 3 patients were alcoholic, 3 patients were smokers, 7 patients had the habit of tobacco chewing, 2 patients had habits in the past, and the remaining patients had 2 or more habits. 22 patients had the routine of eating only vegetarian foods, and 46 patients followed mixed routine in their eating habits.13 patients had only hypertension (HTN), 5 patients had only Type 2 Diabetes mellitus (T2DM), 12 patients had both HTN as well as T2DM, 8 patients had other comorbidities such as preeclampsia, mitral stenosis, hypothyroidism. The majority of the patients were observed without any comorbidities and counted to be 21 in number. NIHSS obtained on the admission of AIS patient showed that almost 13 patients were affected by minor stroke, 45 patients were affected by moderate stroke, 6 patients were found to be in the stroke scale of moderate to severe, and only 8 patients were severely affected.

In distribution among MRSamong the enrolled patients, 12 patients showed no significant disability, 15 patient showed slight disability, 14 patients showed moderate disability, 13 patients showed moderately severe disability, 8 patients showed severe disability and one patient had passed away. Distribution of no. of days patients admitted in hospital results showed that 7 patients had stayed less than 4 days, 41 patients stayed in the range of 5-8 days, 17 patients stayed for 9-12 days, 2 patients stayed for 13-16 days and only one stayed for 18 days. In the Chi-Square analysis of the association between the lifestyle of the patients and their duration of hospital stay, the Pearson chi-square analysis showed significant results $(\mathrm{P}$-value $=0.016<0.05)$.In the Chi-Square analysis of the association between the Lifestyle habits of the Patient and NLA, the Pearson chi-square analysis showed significant results $(\mathrm{P}$-value $=0.049<0.05)$.In the Chi-Square analysis of the association between diet, methods and duration of hospital stay, the Pearson chi-square analysis showed significant results $(\mathrm{P}-\mathrm{value}=0.024<0.05)$. (Table No.1)

In the Chi-Square analysis of the association between, comorbidities and NIHSS, the Pearson chi-square analysis showed significant results $(\mathrm{P}$-value $=0.034<0.05)$.In the Chi-Square analysis of the association between NLR and 
Duration of Hospital Stay, the Pearson chi-square analysis showed significant results(P-value $=0.000<0.001)$.In the Chi-Square analysis for the association between NLR and MRS, the Pearson chi-square analysis showed significant results $(\mathrm{P}$-value $=0.000<0.001)$.In the $\mathrm{Chi}$-Square analysis of the association between NLR and NIHSS, the Pearson chisquare analysis showed that there is no significant association between NLR and NIHSS (P-value $>0.05, \mathrm{P}=0.561$ ). In the Chi-Square analysis of the association between MRS and NIHSS, the Pearson chi-square analysis showed that there is no significant association between NIHSS and Modified ranking scale ( $\mathrm{P}$-value $>0.05, \mathrm{P}=0.474$ ). In the Chi-Square analysis of the association between duration of hospital stay and NIHSS, the Pearson chi-square analysis showed that there is no significant association between NIHSS and duration of hospital stay ( $\mathrm{P}$-value $>0.05, \mathrm{P}=0.561)$. (Table No.2)

Demographic and clinical data were analysed and their frequency and percentage were calculated and shown in the respective table. Using Pearson chi-square analysis, the association between various factors has been analysed and associated statistical significance were interpreted.

At the time of discharge, the functional outcome was calculated using MRS. Results of chi-square analysis inferred the association between NLR and various other clinical factors related to stroke. Analyzing the data showed a significant association between NLR and duration of hospital stay, the lifestyle of the patient as well as the functional outcome at the time of discharge.

\section{DISCUSSION:}

At the time of discharge, the functional outcome was calculated using MRS. Results of chi-square analysis inferred the association between NLR and various other clinical factors related to stroke. Analyzing the data showed a significant association between NLR and duration of hospital stay, the lifestyle of the patient as well as the functional outcome at the time of discharge. Post ischemia, apoptosis brain cells and the tissue release profound inflammatory substances, leading to the accumulation of biomarkers of inflammation like neutrophils, lymphocytes and cytokines. The normal value of NLR in healthy populations was 1.65 (mean), ranging from 0.78 to 3.53. Milena et al considered the relation between NLR and LMR (Lymphocyte to Monocyte ratio) with Ischemic Stroke. 58 adult patients were involved in the study, followed by analysing the blood sample their NLR and LMR were observed. There was no influence of LMR found in the stroke patients. But they found that patients with higher NLR have the possibility of getting a severe stroke. ${ }^{7}$

A study with a large number of patients (1001 patients) carried out for delirium and AIS and its prospective data collection of NLR monitoring. C-reactive protein can control the inflammation, that promotes the neutrophil activation and maintain the NLR value. Patients with higher NLR value involved in the 30-day mortality of AIS patients. ${ }^{8}$

Studies have reported operating outcome is decreased inpatients having higher NL ratio. Disability/Mortality was observed in patients with uninterruptedly elevated NL ratio level at forty-eight hours or more post-ischemic injury in the brain started. Elevated NL ratio is related to both initial and long-term mortality. Elevated NLR levels have increased the possibility of ICH post thrombolysis. In one hundred fiftyone population having primary insult of ischemic brain injury were observed for a month, observations exhibit that the neutrophil-lymphocyte ratio and infarct size correlated. ${ }^{5,9}$

Lymphopenia is another inflammatory marker, which triggers the response to higher production of secondary stress. The inflammatory process carried out with the lymphopenia to promote lymphocyte apoptosis. Lymphopenia has a strong correlation with mortality in patients. The neutrophil count, lymphocyte count, and NLR were obtained from the WBC count, and those values are useable in the prediction of mortality and prognosis of stroke patients and also the markers of systemic inflammation. NLR will be useful in the evaluation of the risk of stroke in asymptomatic patients with plaques causing intermediate carotid artery stenosis. This patient group, with a higher NLR, have a higher chance of stroke. ${ }^{5,9}$

Various clinical research reports mentioned that age, previous stroke history, atrial fibrillation, initial National Institutes of Health Stroke Scale score, and high-sensitivity C-reactive protein has a significant role in the monitoring of NLR. The NLR was higher in the unadorned pneumonia group when it was evaluated by Pneumonia Severity Index. The NLR evaluation helps to identify high-risk patients in the initial and throughout the time of treatment. 116 affected people in the age of 67 show a significant relationship between NLR and $\mathrm{mRS}(\mathrm{P}=0.02)$. In that case report, many of them noted the age-based variation in NLR controlling. NLR is the gladly available biomarker, it is a clinically suitable tool for risk stratification when assessing AIS patients. ${ }^{9,10}$

Another study reported with 293 large vessel occlusion patients, they observed higher median admission NLR values were noted in symptomatic intracranial haemorrhage. Higher admission NLR is a sovereign predictor of symptomatic intracranial haemorrhage and it may recognize a target group for testing adjunctive anti-inflammatory therapies. Major research reports explained that the elevated NL ratio values have been related to the higher incidence of hemorrhagic transformation or SICH in AIS patients getting intravenous thrombolysis.

AIS use the healthy immune system in animal models of the middle cerebral artery for the migration of neutrophils in the brain. One of the individual components of PREDICT scale is admission NLR is the most widely used in the treatment. ${ }^{5}$ 
Post-stroke inflammation has a dual role in ischemic stroke. Peripheral immune cells are getting stimulated post-stroke can in turn affect the fate of brain tissue that has undergone ischemia. Neutrophils expressed early after stroke to promote the active inflammatory reaction, while lymphocytes also having the regulatory function in inflammation persuading neuroprotection. If there is any reduction of neutrophils and induction of lymphocyte after stroke, that can progress functional outcome of AIS. . $^{5,9,10}$

\section{CONCLUSION}

The study suggests that the majority of the patients had moderate stroke outcome and the duration of hospital stay was significantly associated with bad lifestyle and functional outcome at the time of discharge. The rise in NLR helps to identify the high risk of AIS among patients. Hence NLR can be used as a biomarker for risk stratification among AIS patients.

\section{ACKNOWLEDGEMENT}

Authors acknowledge the immense help received from the scholars whose articles are cited and included in references of this manuscript. The authors are also grateful to authors/ editors/publishers of all those articles, journals and books from where the literature for this article has been reviewed and discussed.

\section{Source of Funding: None}

Conflict of Interest: None

Authors' Contribution: All the authors have significantly contributed to manuscript writing, editing and statistical analysis.

\section{REFERENCES}

1. Feigin VL, Roth GA, Naghavi M, Parmar P, Krishnamurthi $\mathrm{R}$, Chugh Set al., Global burden of stroke and risk factors in 188 countries, during 1990-2013: a systematic analysis for the Global Burden of Disease Study 2013. The Lancet Neurol. 2016. 15(9): 913-924.

2. Chung JW, Ryu WS, Kim BJ, Yoon BW, Elevated Calcium after Acute Ischemic Stroke: Association with a Poor Short-Term Outcome and Long-Term Mortality. J Sci. 2015;17(1):54-59.

3. Heit JJ, Sussman ESandWintermark M, Perfusion computed tomography in acute ischemic stroke. Radio Clin. 2019. 57(6): 1109-1116.

4. Leigh R, Knutsson L, Zhou J and Peter CM, Imaging the physiological evolution of the ischemic penumbra in acute ischemic stroke. Radio Clin. 2018. 38(9):1500-1516.

5. Nam KW, Kim TJ, Lee JS, Kwon HM, Lee YS, Ko SB et al., High neutrophil-to-lymphocyte ratio predicts stroke-associated pneumonia. Stroke. 2018. 49(8): 1886-1892.

6. Lee JH, Kwon KY, Yoon SY, Kim HS, and Lim CS, Characteristics of platelet indices, neutrophil-to-lymphocyte ratio and erythrocyte sedimentation rate compared with $\mathrm{C}$ reactive protein in patients with cerebral infarction: a retrospective analysis of comparing haematological parameters and $\mathrm{C}$ reactive protein. BMJ Open. 2014. 4(11): 182.

7. Segatore, M, Understanding central post-stroke pain. JNN, 1996. 28(1): 28-35.

8. Kotfis K, Bott-Olejnik M, Szylińska A, Rotter I, Could Neutrophil-to-Lymphocyte Ratio (NLR) serve as a potential marker for delirium prediction in patients with acute ischemic stroke? A Prospective Observational Study. J Clin Med. 2019. 8(7): 1075.

9. Yu S, Arima H, Bertmar C, Clarke S, Herkes G, Krause M et al. Neutrophil to lymphocyte ratio and early clinical outcomes in patients with acute ischemic stroke. J Neurol. 2018. 387: 115118.

10. Zhang J, Ren Q, Song, Y, He M, Zeng Y, Liu Z, Xu J et al. Prognostic role of neutrophil-lymphocyte ratio in patients with acute ischemic stroke. J Med. 2017; 96(45): e8624.

Table 1: Lifestyle and NLR factors associated with duration of Hospital stay

\begin{tabular}{|c|c|c|c|c|c|c|c|}
\hline & & \multicolumn{5}{|c|}{ Duration of Hospital stay } & \multirow{2}{*}{$\begin{array}{l}\text { Pearson Chi- } \\
\text { Square, Df, P }\end{array}$} \\
\hline & & $<=4$ days & 5-8 days & 9-12 days & 13-16 days & 17-20 days & \\
\hline \multirow{7}{*}{$\begin{array}{l}\text { Lifestyle } \\
\text { habits of } \\
\text { patient }\end{array}$} & $\begin{array}{l}\text { Without smoking/ } \\
\text { drinking/TC }\end{array}$ & 6 & 31 & 11 & 1 & 1 & \multirow{7}{*}{$41.130,24, .016$} \\
\hline & Alcoholic & o & 2 & 1 & o & o & \\
\hline & Smoker & o & 2 & 1 & o & o & \\
\hline & Tobacco Chewer & o & 4 & 3 & o & o & \\
\hline & $\begin{array}{l}\text { Alcoholic with TC/ } \\
\text { Smoker }\end{array}$ & o & 1 & 1 & o & o & \\
\hline & $\begin{array}{l}\text { Habits in the past or } \\
\text { occasional }\end{array}$ & 1 & 1 & o & o & o & \\
\hline & $\begin{array}{l}\text { Smoker, Alcoholic and } \\
\text { TC }\end{array}$ & o & o & o & 1 & o & \\
\hline
\end{tabular}


Table 1: (Continued)

\begin{tabular}{|c|c|c|c|c|c|c|c|}
\hline & & \multicolumn{5}{|c|}{ Duration of Hospital stay } & \multirow{2}{*}{$\begin{array}{l}\text { Pearson Chi- } \\
\text { Square, Df, P }\end{array}$} \\
\hline & & $<=4$ days & 5-8 days & 9-12 days & 13-16 days & 17-20 days & \\
\hline \multirow[t]{2}{*}{ Diet } & Vegetarian & 5 & 14 & 2 & o & 1 & $\begin{array}{l}11.284 \\
4, .024\end{array}$ \\
\hline & Mixed & 2 & 27 & 15 & 2 & o & \\
\hline \multirow[t]{5}{*}{ NLR } & Normal NLR & 3 & 7 & 2 & o & o & $46.205,16, \mathbf{0 . 0 0 0}$ \\
\hline & Possibility of Stroke & 4 & 21 & 4 & o & o & \\
\hline & Mild Stroke & o & 8 & 7 & 1 & o & \\
\hline & Moderate Stroke & o & 5 & 3 & o & o & \\
\hline & Severe Stroke & o & o & 1 & 1 & 1 & \\
\hline
\end{tabular}

Table 2: Association of NLR with level of stroke and disability

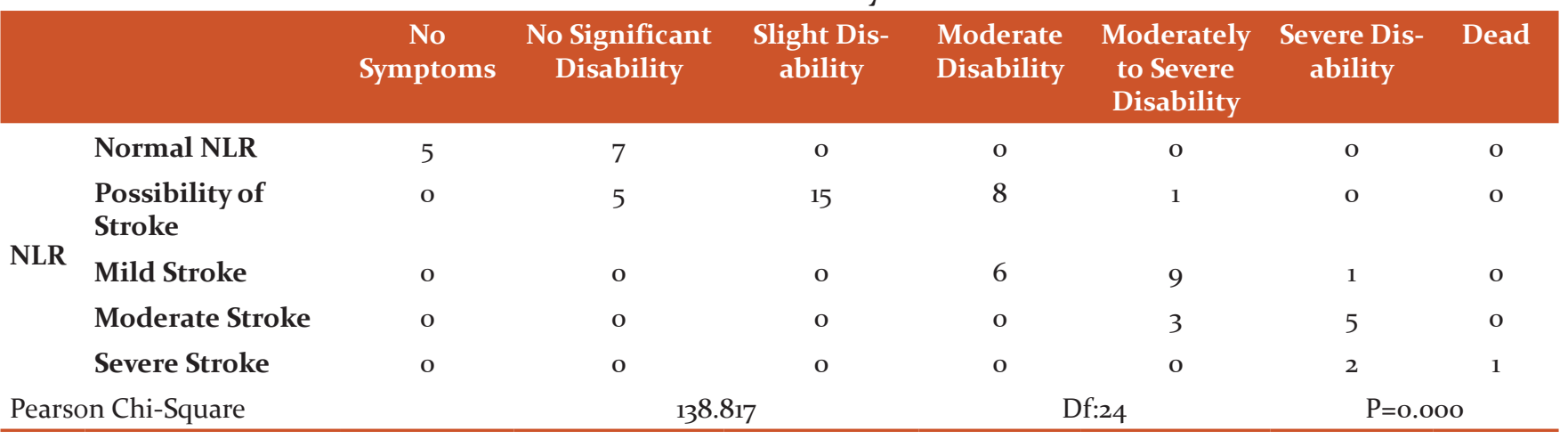

